\title{
Dudosa relación entre exposición a la televisión y los videojuegos y el desarrollo de problemas de atención en niños y adolescentes
}

Doubtful association between exposure to television and video games, and the development of attention problems in children and adolescents

Edward L. y col. Pediatrics 2010;126:214-21.

\section{Objetivos}

Evaluar si la exposición a la televisión (TV) y los videojuegos (VJ) se asocia con problemas de atención (PA) en niños y adolescentes.

\section{Diseño, lugar y pacientes}

Estudio observacional mixto realizado en EE.UU. que incluyó una cohorte de 1.323 niños (edad media 9,6 años) provenientes de diez escuelas primarias, y una muestra de 210 estudiantes universitarios (edad media 19,8 años).

\section{Intervención}

La cohorte de niños fue evaluada en cuatro instancias durante 13 meses. Para esto se utilizaron auto-reportes (completados por los niños y sus padres) que recogieron información sobre cantidad de horas semanales de exposición a TV y VJ, y también un informe de la maestra que evaluó el comportamiento en el aula medido a través de tres ítems que evaluaban PA utilizando una escala tipo Likert* de cinco puntos.

Los adolescentes fueron evaluados en un único tiempo a través de un auto-reporte de exposición a TV y VJ, y tres cuestionarios validados para detección de PA.

\section{Medición de resultados principales}

Se analizó la asociación entre los PA y exposición a TV y VJ mediante el calculo de coeficientes de correlación*. Para analizar los cambios en el tiempo de los PA en la cohorte de niños se utilizaron modelos lineales ${ }^{*}$ usando como covariables: género, año escolar y los PA al comienzo del estudio.

\section{Resultados principales}

Los coeficientes de correlación entre exposición y PA fueron pequeños a moderados en ambas muestras de pacientes ( $r 0,23$ y 0,27). Tomando la recomendación de la Academia Americana de Pediatría (AAP) de no exponer a los niños más de dos horas diarias a TV y VJ y con los datos obtenidos previamente, se calculó a través de regresiones logísticas ${ }^{\star}$ el riesgo superar la mediana de PA en los niños y adolescentes con exposiciones por encima y por debajo de dichas recomendaciones. Los que superaron la cantidad de horas recomendadas mostraron ser más propensos a estar por encima de la mediana en los PA: niños, OR 1,67 (IC 95\% 1,27 a 2,21); adolescentes, OR $2,23(1,13$ a 4,39$)$. Los modelos lineales mostraron en la cohorte de niños que presentar PA al comienzo del seguimiento, ser varón, y cursar grados inferiores eran predictores de PA al final del seguimiento.

\section{Conclusiones}

Tanto la exposición a televisión como a videojuegos se asocian a incrementos subsecuentes de problemas de atención en niños. Pareciera haber una asociación similar en adolescentes.

Fuente de Financiamiento: No referida

\section{Comentario}

Para poder responder las preguntas planteadas por los investigadores con una buena aproximación de temporalidad, el estudio mas apropiado hubiese sido una cohorte prospectiva en la cual se realice una prueba basal para descartar PA en los grupos, seguida de una medición posterior del evento de interés luego de ocurrida la exposición a TV y VJ. Otra opción podría haber sido un estudio de casos-controles.

Medir los PA en niños con un cuestionario basado solo en tres ítems no parece suficiente. Siendo esta la información más importante que requiere el estudio para contestar su pregunta, se podría haber utilizado otro método más preciso en lugar del reporte de la maestra. Las conclusiones basadas en la comparación con la mediana de los PA no tienen mucho significado clínico, más interesante y útil hubiese sido medir algún parámetro clínico que evalúe PA significativos, o datos que muestren a cuales de los niños les va peor, por ejemplo, en su rendimiento escolar.

Los coeficientes de correlación que se reportan como pequeños a moderados en realidad son insignificantes y no muestran relación entre la exposición y los PA.

\section{Conclusiones del comentador}

El estudio está lejos de responder sus preguntas ya que tiene poca validez interna. El haber reportado conclusiones tan firmes con la metodología utilizada puede confundir al lector no entrenado en trabajos científicos, y promover innecesariamente el cambio de conductas cotidianas sin demostración de que estas sean riesgosas, por lo menos hasta el momento y en lo que refiere a los problemas de atención.

Yamila De Vas [ Servicio de Medicina Familiar y Comunitaria del Hospital Italiano de Buenos Aires yamila.devas@hospitalitaliano.org.ar ]

De Vas Yamila. La exposición a la televisión y los videojuegos se asocia a mayores problemas de atención en nilos Evid Act Pract Ambul. Abr-Jun 2012;15(2):55 Comentado de Edward L. Swing, MS, Douglas A. y col. Television and Video Game Exposure and the Development of Attention Problems. Pediatrics 2010;126:214-21. PMID: 20603258. 